\title{
Epidural angiomatous meningioma of the thoracic spine: A case report
}

\author{
TAO YANG, LIANG WU, CHENLONG YANG and YULUN XU \\ Department of Neurosurgery, China National Clinical Research Center for Neurological Diseases, \\ Beijing Tiantan Hospital, Capital Medical University, Dongcheng, Beijing 100050, P.R. China
}

Received October 25, 2014; Accepted August 6, 2015

DOI: 10.3892/ol.2015.3869

\begin{abstract}
Spinal epidural angiomatous meningiomas (AMs) are extremely rare lesions. Here, we report on a case of an epidural AM of the thoracic spine with chronic but severe cord compression. The patient underwent a T6-T8 laminectomy through the posterior approach. En bloc resection was achieved, and histopathological examination demonstrated an AM. Delayed paraplegia occurred $4 \mathrm{~h}$ postoperatively. The patient was treated with methylprednisolone, hyperbaric oxygen and rehabilitation. Gradually, over the next six months, the bilateral leg strength was improved compared with the preoperative status, and no tumor recurrence was noted. Although epidural AM is extremely rare, it should be included in the differential diagnosis of spinal epidural lesions. A definitive diagnosis is difficult based on magnetic resonance imaging alone due to the nonspecific characteristics of the tumor. Since AM is a histologically benign and highly vascularized tumor, timely gross total resection (GTR) is the most effective treatment. A good clinical outcome may be expected following GTR (Simpson grade I and II resection).
\end{abstract}

\section{Introduction}

Angiomatous meningiomas (AMs) are rare histopathological subtypes of meningiomas, and are most commonly observed within the cerebral convexity (1). Microscopically, the lesions have features of typical meningiomas, with numerous vascular channels which predominate over their meningothelial elements $(2,3)$. AMs also occasionally occur in the spinal canal, and account for $1 \%$ of all intraspinal meningiomas (2). The majority of spinal AMs are located in the intradural-extramedullary (IDEM) space $(4,5)$. Compared with other IDEM tumors, intraspinal AMs have no unique clinical

Correspondence to: Dr Yulun Xu, Department of Neurosurgery, China National Clinical Research Center for Neurological Diseases, Beijing Tiantan Hospital, Capital Medical University, 6 Tiantan Xili, Dongcheng, Beijing 100050, P.R. China

E-mail: xuhuxi@sina.cn

Key words: angiomatous meningioma, epidural, spinal symptoms (2). Instead, the diagnosis depends on pathological examination (3). Epithelial membrane antigen (EMA) is the most reliable immunomarker and exhibits positive immunoreactivity in all AMs $(2,3)$. Timely en bloc resection is the most favorable treatment strategy and the rate of tumor recurrence is fairly low, ranging between 0 and $60 \%(2,4,5)$. Epidural AMs are extremely rare. In the present study, we report the case of an epidural AM in a 55-year-old female. The clinical manifestation, radiological findings, treatment and outcome of the patient are presented. Written informed consent was obtained from the patient's family and study approval was obtained from the Institutional Review Board of Beijing Tiantan Hospital, Beijing, China.

\section{Case report}

A 55-year-old female presented at Beijing Tiantan Hospital, China, with a 2-year history of progressive back pain and weakness in both legs. No sphincter dysfunction was noted. Neurological examination revealed a paraparesis, with motor deficit 3/5 in the both legs (as classified by the Medical Research Council grading system) (6). This finding was associated with hypesthesia and brisk osteotendinous reflexes.

Magnetic resonance imaging (MRI) demonstrated a well-circumscribed oval lesion in the dorsal-lateral space at T6-8 (Fig. 1). The lesion was isointense on T1-weighted images (WI), hyperintense on T2-WI, and enhanced homogeneously on contrast-enhanced T1-WI. The 'dural tail sign' was positive. The preoperative differential diagnoses considered included lymphoma, metastatic tumor, angiolipoma, cavernous hemangioma, meningioma and hemangiopericytoma (HPC).

The patient underwent a T6-8 laminectomy. Intraoperative monitoring of somatosensory and motor-evoked potentials was performed. The lesion was well-delineated, fresh red, vascular, and located in the epidural space. It was removed en bloc following circumferential dissection. The dural attachment was located dorsal-laterally and was not resected but extensively bipolar cauterized. Postoperative MRI revealed no residual tumor (Fig. 2).

Microscopically, the tumor consisted of meningothelial and vascular elements (Fig. 3). There was no mitotic activity or endothelial proliferation. Immunohistochemical examination revealed that the tumor cells were positive for EMA and vimentin. 
A

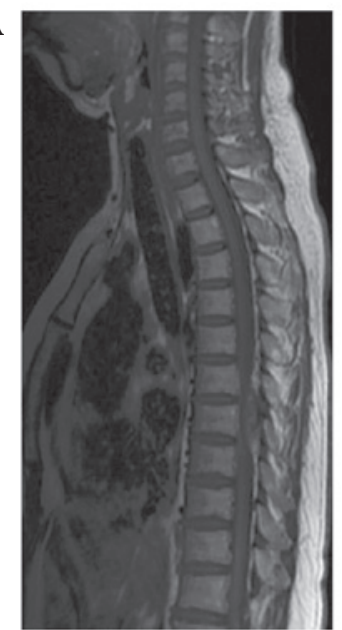

B

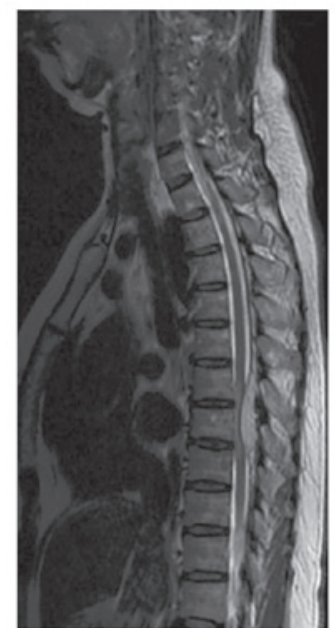

C

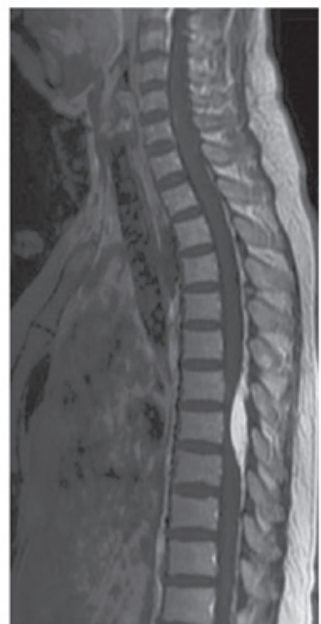

D

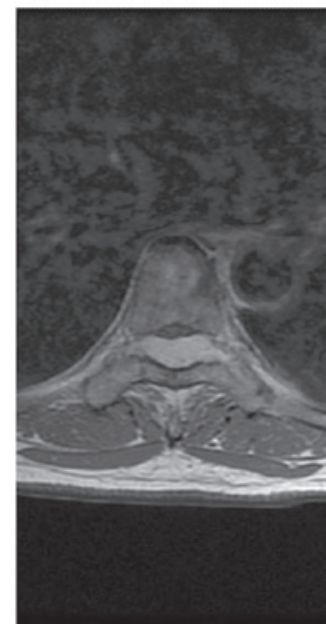

Figure 1. Magnetic resonance imaging reveals a well-defined epidural tumor at the T6-T8 level. The tumor was isointense on T1-weighted imaging (WI) (A) and hyperintense on T2-WI (B). Marked homogeneous enhancement and the 'dural tail sign' were observed on contrast-enhanced T1-WI (C). Axial contrast-enhanced T1-WI revealed that the tumor was located dorsal-laterally, with severe cord compression and extension through the intervertebral foramen (D).

A

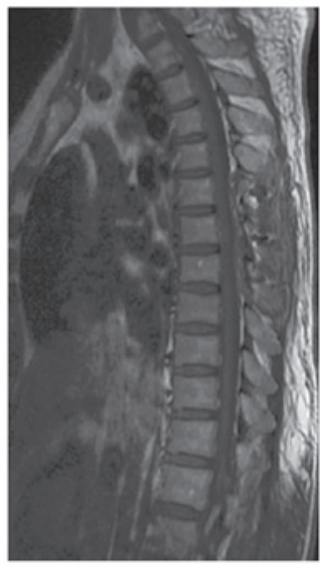

B

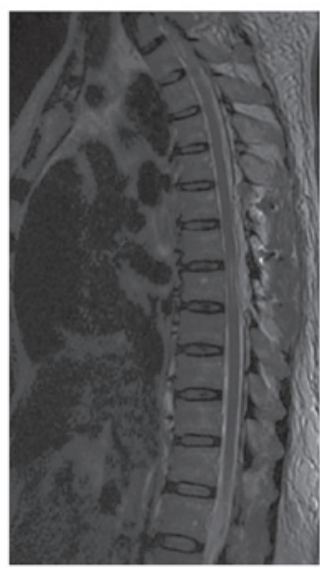

C

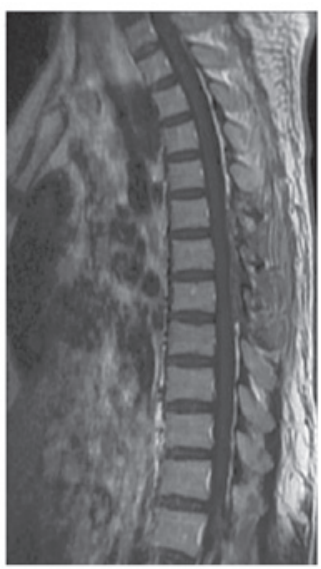

D

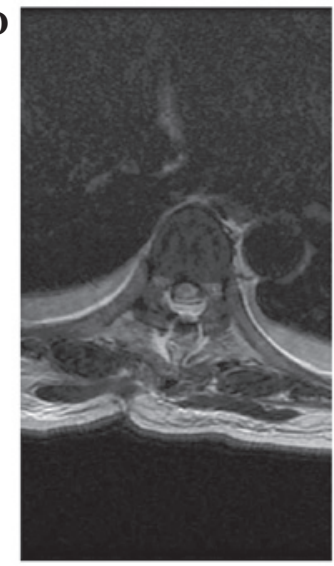

Figure 2. Postoperative magnetic resonance imaging reveals no residual tumor or intraspinal hematoma, and the spinal cord is decompressed. (A) T1-weighted image (WI), (B) T2-WI, (C) contrast-enhanced T1-WI, (D) axial T2-WI.

A

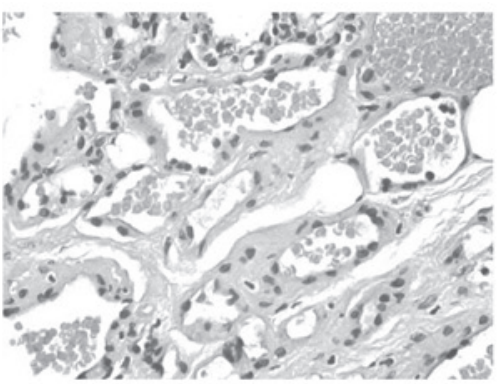

B

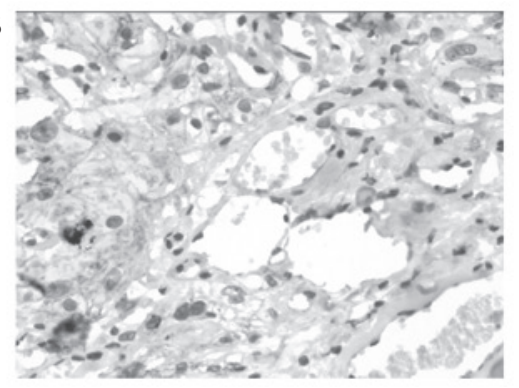

C

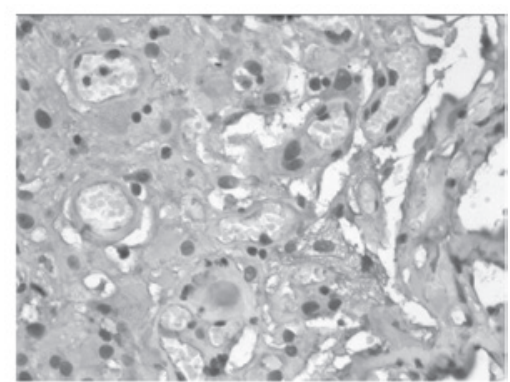

Figure 3. Photomicrographs illustrate that the tumor consisted of dilated vascular spaces with intervening areas revealing spindle and oval cells. (A) No evidence of endothelial proliferation or mitotic activity was observed (hematoxylin and eosin staining; original magnification, x200). (B and C) Tumor cells were positive for (B) epithelial membrane antigen and (C) vimentin (immunohistochemical stain; original magnification, x200).

Delayed paraplegia occurred at $4 \mathrm{~h}$ postoperatively. The patient was treated with methylprednisolone and hyperbaric oxygen, and was discharged to an in-patient rehabilitation facility at day 14 postoperatively. Gradually, over the next six months, the patient's bilateral leg strength improved to grade $4 / 5$, and no tumor recurrence was noted.

\section{Discussion}

Spinal meningiomas are mostly IDEM in location, and the diagnosis of a tumor is usually simple, and based on radiological findings. However, meningiomas also occasionally occur in an epidural location. Since the publication of the 
earliest study in 1963, only 50 cases of epidural meningiomas have been described, and psammomatous types were the predominant subtype (7-10). Epidural AMs are extremely rare. No other examples of foraminal extension of this specific type were identified in the literature.

The precise pathogenesis of epidural meningiomas is still unclear. Various hypotheses have been proposed, which include arachnoid villi isolated from the arachnoid layer which migrated into the dura mater, vestigial remnants of the embryonal arachnoid layer, aberrant arachnoid islets in the epidural space, and villi from nerve roots where the dura layer is thin $(7,8,11,12)$.

Similar to those observed with common epidural tumors, the symptoms of our patient were progressive motor deficits and local pain due to the effect of the mass. The clinical course was relatively slow (24 months), which may reflect the benign nature of epidural AMs. Unlike epidural cavernous hemangioma, which may present with acute neurological symptoms due to their tendency to hemorrhage (13), epidural AM does not appear to have a propensity for acute intratumoral bleeding despite being highly vascularized. Similarly, spontaneous intratumoral bleeding was only observed in one intracranial AM (2). This phenomenon argues against a high incidence of hemorrhage in AMs.

MRI is the most reliable method for identifying intraspinal tumors. On MRI, the epidural AM in the present case demonstrated isointensity on T1-WI, hyperintensity on T2-WI, and homogeneous enhancement on contrast-enhanced T1-WI. Intracranial AMs usually exhibit notable signal voids of vessels (1); however, signal void areas were not evident in the present case. These radiological characteristics are nonspecific. The 'dural tail sign', which is useful for the identification of IDEM meningioma, was positive in the current case. However, this finding has also been present in metastatic tumors and lymphoma $(14,15)$. Thus, the differential diagnosis includes all epidural contrast-enhancing lesions, including lymphoma, metastatic tumor, cavernous hemangioma, angiolipoma and HPC. An accurate diagnosis depends on the pathology. However, due to the similar microscopic features, hematoxylin and eosin staining may be not sufficient to differentiate AMs from HPCs. Therefore, immunohistochemical staining is necessary. EMA is the most reliable immuno-marker of meningiomas, whereas HPCs are negative with EMA $(16,17)$. In the current case, the tumor exhibited abundant vascular components which exceeded $50 \%$ of the total area, a lack of cytonuclear atypia or mitotic activity, and EMA immunoreactivity. Thus, the tumor was diagnosed as AM (World Health Organization grade I).

Since cord compression is the main pathogenic mechanism, gross total resection (GTR) is the primary treatment of choice for spinal lesions $(18,19)$. Due to the hypervascularity, piecemeal resection of AM should be avoided (19). In the present case, the feeding arteries were coagulated and en bloc removal was performed. Selective spinal angiography and preoperative embolization may exhibit feeding arteries and reduce intraoperative bleeding (1); however, the diagnosis still needs to be confirmed. For intracranial and intraspinal meningiomas, tumor recurrence may be prevented by total removal of the tumor and dural attachment $(1,2)$. However, if the dural attachment of intraspinal meningiomas is located ventrally, laterally or dorsal-laterally, cauterizing the dura takes priority over dural resection, preventing postoperative cerebrospinal fluid leak or cord damage (4). Although the neurological function of our patient was deteriorated following surgery, the symptoms were improved to normal levels within six months, and no tumor recurrence was noted. Therefore, epidural AMs are amenable to surgery, and a positive clinical outcome following GTR (Simpson grade I and II resection) is expected.

In conclusion, AM should be included in the differential diagnosis of spinal epidural lesions. A definitive diagnosis is difficult based on MRI alone due to its nonspecific characteristics. Since AM is a histologically benign and highly vascularized tumor, timely GTR (en bloc resection) is the most effective treatment. A good clinical outcome may be expected following GTR (Simpson grade I and II resection).

\section{References}

1. Liu Z, Wang C, Wang H, Wang Y, Li JY and Liu Y: Clinical characteristics and treatment of angiomatous meningiomas: a report of 27 cases. Int J Clin Exp Pathol 6: 695-702, 2013.

2. Hasselblatt M, Nolte KW and Paulus W: Angiomatous meningioma: a clinicopathologic study of 38 cases. Am J Surg Pathol 28: 390-393, 2004

3. Levy WJ Jr, Bay J and Dohn D: Spinal cord meningioma. J Neurosurg 57: 804-812, 1982.

4. Boström A, Bürgel U, Reinacher P, Krings $T$, Rohde V, Gilsbach JM and Hans FJ: A less invasive surgical concept for the resection of spinal meningiomas. Acta Neurochir (Wien) 150: 551-556, 2008.

5. Nakamura M, Tsuji O, Fujiyoshi K, Hosogane N, Watanabe K, Tsuji T, Ishii K, Toyama Y, Chiba K and Matsumoto M: Long-term surgical outcomes of spinal meningiomas. Spine (Phila Pa 1976) 37: E617-E623, 2012.

6. Dyck PJ, Boes CJ, Mulder D, Millikan C, Windebank AJ, Dyck PJ and Espinosa R: History of standard scoring, notation and summation of neuromuscular signs. A current survey and recommendation. J Peripher Nerv Syst 10: 158-173, 2005.

7. Haft $\mathrm{H}$ and Shenkin Ha: Spinal epidural meningioma: Case report. J Neurosurg 20: 801-804, 1963.

8. Fortuna A, Gambacorta D and Occhipinti EM: Spinal extradural meningiomas. Neurochirurgia (Stuttg) 12: 166-180, 1969.

9. Takeuchi H, Kubota T, Sato K and Hirose S: Cervical extradural meningioma with rapidly progressive myelopathy. J Clin Neurosci 13: 397-400, 2006.

10. Zevgaridis D and Thomé C: Purely epidural spinal meningioma mimicking metastatic tumor: case report and review of the literature. Spine (Phila Pa 1976) 27: E403-E405, 2002.

11. Kumar S, Kaza RC, Maitra TK and Chandra M: Extradural spinal meningioma arising from a nerve root: case report. J Neurosurg 52: 728-729, 1980.

12. Sato N and Sze G: Extradural spinal meningioma: MRI. Neuroradiology 39: 450-452, 1997.

13. Sarikaya-Seiwert S, Gierga K, Wessalowski R, Steiger HJ and Hänggi D: Solitary spinal epidural cavernous angiomas in children presenting with acute neurological symptoms caused by hemorrhage. J Neurosurg Pediatr 5: 89-93, 2010.

14. Rokni-Yazdi H and Sotoudeh H: Prevalence of 'dural tail sign' in patients with different intracranial pathologies. Eur J Radiol 60: 42-45, 2006.

15. Tien RD, Yang PJ and Chu PK: 'Dural tail sign': a specific MR sign for meningioma? J Comput Assist Tomogr 15: 64-66, 1991.

16. Rao S, Rajkumar A and Kuruvilla S: Angiomatous meningioma: a diagnostic dilemma. Indian J Pathol Microbiol 51: 53-55, 2008.

17. D'Amore ES, Manivel JC and Sung JH: Soft-tissue and meningeal hemangiopericytomas: an immunohistochemical and ultrastructural study. Hum Pathol 21: 414-423, 1990.

18. Orchowski J, Bridwell KH and Lenke LG: Neurological deficit from a purely vascular etiology after unilateral vessel ligation during anterior thoracolumbar fusion of the spine. Spine (Phila Pa 1976) 30: 406-410, 2005.

19. Wu L, Yang T, Yang C, Deng X, Fang J and Xu Y: Surgical treatment of intraspinal angiomatous meningiomas from a single center. Neurol Med Chir (Tokyo) 55: 328-335, 2015. 